\title{
A Recurrent Neural Network with Non-gesture Rejection Model for Recognizing Gestures with Smartphone Sensors
}

\author{
Myeong-Chun Lee and Sung-Bae Cho \\ Dept. of Computer Science, Yonsei University \\ 50 Yonsei-ro, Seodaemoon-gu, Seoul 120-749, Korea \\ lmspring@sclab.yonsei.ac.kr, sbcho@cs.yonsei.ac.kr
}

\begin{abstract}
Gesture recognition provides a new interface to user. Various methods for the gesture recognition are feasible in smartphone environment since a number of sensors attached are gradually increasing. In this paper, we propose a gesture recognition method using smartphone accelerometer sensors. The high false-positive rate is definite if the gesture sequence data are increased. We have modified BLSTM (Bidirectional Long Short-Term Memory) recurrent neural network with non-gesture rejection model to deal with the problem. A BLSTM model classifies the input into the gesture and non-gesture classes, and the specific BLSTM models for the gestures further classify it into one of twenty gestures. 24,850 sequence data are used for the experiment, and it consists of 11,885 gesture sequences and 12,965 non-gesture sequences. The proposed method shows higher accuracy than the standard BLSTM.
\end{abstract}

Keywords: Smartphone Accelerometer Sensors, Gesture Spotting, Gesture Recognition, Recurrent Neural Network.

\section{Introduction}

Recently, various sensors such as accelerometer, ambient light, proximity, dual cameras, GPS, dual microphones, compass, and gyroscope are attached in a smart phone. Those not only are sophisticated but also show good performance. It is possible to recognize the user's pattern or aware the user's intention from the smartphone sensors. Especially, accelerometer is one of the most commonly used sensors for the physical movements of the user bringing the phone together. For this reason, much user interface works such as gesture recognition, and activity recognition are proposed [1].

However, there are two crucial problems when user recognition systems are developed from the smartphone sensors. One is the recognition of the non-gesture or nonactivity data. The data for gesture or activity consist of meaningful and non-meaningful parts. Non-meaningful data can be half of total data or even more than gesture data. In this case, it is time-consuming to recognize both meaningful and non-meaningful data. The other is to maintain a high-accuracy rate even though the number of classes is increased. Most works about pattern recognition use machine learning methods to learn 
the data. However, the performance degrades when classifying a large number of classes. It is the important problem when supporting the various services to users.

In this paper, we propose a mobile gesture recognition method where data is collected from an accelerometer sensor attached to smartphone. To alleviate aforementioned two problems, a recurrent neural network called BLSTM (Bidirectional Long Short-Term Memory) is used for classifying twenty gestures. To distinguish the gesture and non-gesture, a non-gesture rejection model is proposed.

\section{Related Works}

The relevant research about gesture recognition is divided into several groups. One is the vision-based gesture recognition. Q. Chen et al. proposed a hand gesture recognition method with the combination of statistical and syntactic analyses. They used a web camera as the input device and constructed the system for real time environment [2]. Tran et al. suggested a 3-D posture tracking for real time hand gestures. They used nearest neighbor clustering algorithm based on LCS (Longest Common Subsequence) similarity measure of joint angle dynamics [3]. Next group of methods is to recognize gestures based on EMG (Electromyogram) sensor, which is a device to measure the electrical potentials generated by muscle cells. Zhang et al. used both accelerometer and EMG to classify 72 Chinese Sign Language (CSL) [4]. Wheeler et al. utilized the EMG to recognize their arm movement to control a joystick. The Bayesian decomposition method is proposed to distinguish individual muscle groups with the goal of enhancing gesture recognition [5].

Another method recognizes gestures based on accelerometer. It is divided into two groups again, using accelerometer chip only and using the accelerometer attached in a smartphone. Xu et al. used MEMS 3-axes accelerometer for seven hand gestures with the Hopfield network as associative memory for classification algorithm. A decision tree and multi-stream HMMs (Hidden Markov Models) were utilized as decisionlevel fusion to get classification result [6]. Each method has merits and demerits. In case of the vision-based approach, it is possible to recognize the multiple objects. However, it has a limitation to record data because of the constraints of the camera placement. The detailed data can be collected from EMG but the data cannot be collected wirelessly. Accelerometer is more suitable device to collect the gesture data since it is used without the constraints of place and can be collected the gesture data accurately. For our problem, we use an accelerometer in a smartphone. The related works using accelerometer are shown in Table 1.

Table 1. Related Workd using Mobile Accelerometer

\begin{tabular}{|c|c|c|c|c|c|}
\hline Author & Year & Classifier & Gestures & Collector & Overview \\
\hline Liu et al. [7] & 2009 & DTW & 8 & Smartphone & $\begin{array}{c}\text { Personalized gesture using } \\
\text { uWave }\end{array}$ \\
\hline Niezen et al. [8] & 2009 & $\begin{array}{c}\text { HMM, ANN, } \\
\text { DTW }\end{array}$ & 8 & Smartphone & $\begin{array}{c}\text { Performance comparison be- } \\
\text { tween many classifiers }\end{array}$ \\
\hline
\end{tabular}


Table 1. (Continued)

\begin{tabular}{|c|c|c|c|c|c|}
\hline Author & Year & Classifier & Gestures & Collector & Overview \\
\hline Min et al. [9] & 2010 & $\begin{array}{c}\text { DTW, NB, K- } \\
\text { means }\end{array}$ & 20 & Smartphone & $\begin{array}{c}\text { DTW model selection } \\
\text { through NB }\end{array}$ \\
\hline Marasovic et al. [10] & 2011 & K-NN & 7 & Smartphone & $\begin{array}{c}\text { Combination of the } \\
\text { PCA and K-NN }\end{array}$ \\
\hline Akl et al. [11] & 2011 & DTW, AP & 18 & Wii mote & $\begin{array}{c}\text { Dimensionality reduction } \\
\text { through RP }\end{array}$ \\
\hline
\end{tabular}

Inputdata
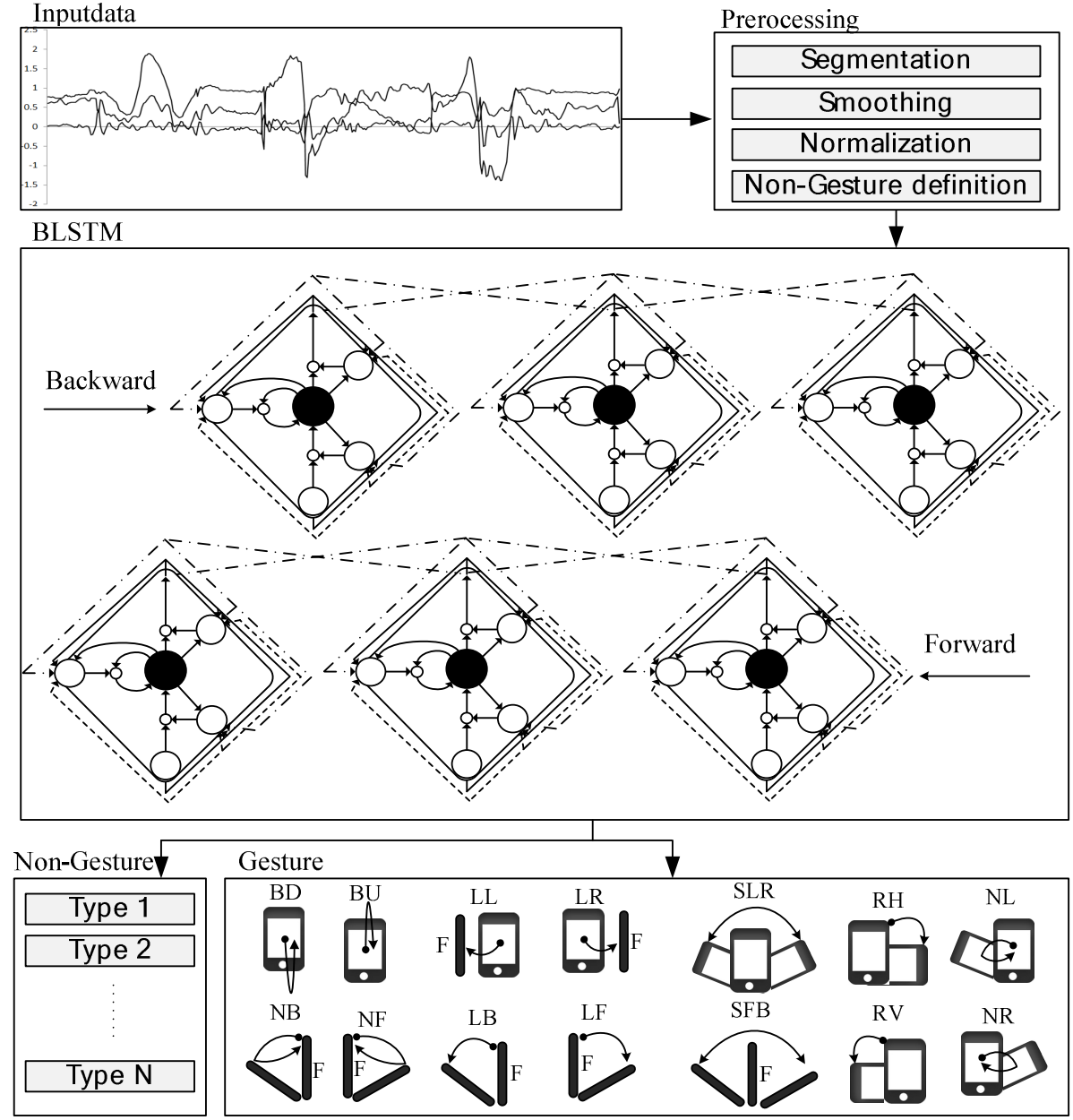

Fig. 1. Overview of the proposed method 


\section{The Proposed Method}

This paper aims to enhance the accuracy by using non-gesture rejection model. The entire system configuration is shown in Fig 1. The accelerometer data collected from a smart phone are segmented by using sliding window and average variation. The preprocessed data are classified after training. We adopt the recurrent neural network based on BLSTM which is a hybridization of BRNN (bidirectional recurrent neural network) and LSTM. A set of training data is used to classify the gestures and nongestures, and the other set of training data for classifying the twenty gesture classes.

LSTM is an extension of the recurrent neural network. It uses the three gates that can store and access the data collected from the rest of the network. The gates are activated from logistic sigmoid activation function. The performance outweighs HMM used for time-series classification problem. The Hyperbolic tangent activation function is used for squashing functions. The basic calculation of each gate is the same with standard artificial neural network [12].

The input gate determines whether the input values put the memory cell or not.

$$
\alpha_{\iota}^{t}=\sum_{i=1}^{I} w_{i \iota} x_{i}^{t}+\sum_{\substack{h=1 \\ \beta_{\iota}^{t}=f\left(\alpha_{\iota}^{t}\right)}}^{H} w_{h \iota} \beta_{h}^{t-1}+\sum_{c=1}^{C} w_{c l} s_{c}^{t-1}
$$

where $\alpha_{\iota}^{t}$ is a state of the input gate at time t. It is calculated from input values, the output of other networks, and state of the memory cell. I, H, and C mean the numbers of input node, hidden node, and cell, respectively. $\mathrm{w}$ is the weight of connected nodes. $f(x)$ is the logistic sigmoid function to activate the input gate.

The output gate determines whether the information is output or not. The calculation of the output gate is similar with the input gate.

$$
\alpha_{\Phi}^{t}=\sum_{i=1}^{I} w_{i \Phi} x_{i}^{t}+\sum_{\substack{h=1 \\ \beta_{\Phi}^{t}=f\left(\alpha_{\Phi}^{t}\right)}}^{H} w_{h \Phi} \beta_{h}^{t-1}+\sum_{c=1}^{C} w_{c \Phi} s_{c}^{t-1}
$$

where $\alpha_{\Phi}^{t}$ is a state of the forget gate at time t and $\beta_{\Phi}^{t}$ is a state after applying the activation function.

Eq. (3) is a calculation that is generated by forget gate, the state of cell, the state of input gate, and state of $\alpha_{c}^{t}$ after applying hyperbolic tangent activation function. Note that the fixed weight value 1.0 is used for preserving the information in the memory cell.

$$
\begin{gathered}
\alpha_{c}^{t}=\sum_{\substack{i=1 \\
\mathrm{~S}_{c}^{t}=\beta_{\Phi}^{t} \mathrm{~S}_{c}^{t-1}+\beta_{l}^{t} \mathrm{~g}\left(\alpha_{c}^{t}\right)}}^{I} x_{i}^{t}+\sum_{h c}^{H} w_{h} \beta_{h}-1
\end{gathered}
$$

Forget gate provides the information to reset the memory cell.

$$
\alpha_{\omega}^{t}=\sum_{i=1}^{I} w_{i \omega} \alpha x_{i}^{t}+\sum_{\substack{h=1 \\ \beta_{\omega}^{t}=f\left(\alpha_{\omega}^{t}\right)}}^{H} w_{h \omega} \beta_{h}^{t-1}+\sum_{c=1}^{C} w_{c \omega} s_{c}^{t-1}
$$


where $\omega$ is the output gate and $\beta_{\omega}^{t}$ is the state of an output gate after applying the activation function at time $\mathrm{t}$.

Eq. (5) is the definition of the cell output. To activate the cells, hyperbolic tangent is used and multiplies the state of output gate.

$$
\beta_{c}^{t}=\beta_{\omega}^{t} \mathrm{e}\left(s_{c}^{t}\right)
$$

For training the LSTM recurrent neural network, we use the Back Propagation Through Time (BPTT) algorithm.

\section{Experimental Results}

For the experiments, Samsung Omnia smartphone is used with MS Windows Mobile 6.1 as a platform. The acceleration is sampled at 50Hz. 30 people of 10 60 years old participate in the experiments. The collected data are divided into generations and date. Total amount of the data consists of 11,885 gesture sequences and 12,965 nongesture sequences. The number of files which is used in the experiment is 1,075.

Table 2 shows the symbol of twenty gestures, direction and meaning. Rotating and tilting to hold their physical states after the movement. Tapping represents the hand or finger stroke on a smart phone surface. In the case of shaking, subjects shake the devices two or more times in a specific direction. Snapping has an angular acceleration while bouncing moves straightly to a direction and reflected back where both are the kind of pendulum movement.

Table 2. Types of Gesture Classes

\begin{tabular}{|c|c|c|c|c|c|}
\hline Symbol & Direction & Meaning & Symbol & Direction & Meaning \\
\hline $\mathrm{NL}$ & Left & \multirow{4}{*}{ Snapping } & TL & Left & \multirow{6}{*}{ Tapping } \\
\hline NR & Right & & TR & Right & \\
\hline $\mathrm{NF}$ & Forward & & TF & Forward & \\
\hline NB & Backward & & TB & Backward & \\
\hline $\mathrm{BU}$ & Up & \multirow{2}{*}{ Bouncing } & TT & Top & \\
\hline $\mathrm{BD}$ & Down & & TM & Bottom & \\
\hline LL & Left & \multirow{4}{*}{ Tilting } & RH & Horizontal & \multirow{2}{*}{ Rotating } \\
\hline LR & Right & & RV & Vertical & \\
\hline $\mathrm{LF}$ & Forward & & SLR & Left-Right & \multirow[b]{2}{*}{ Shaking } \\
\hline LB & Backward & & SFB & $\begin{array}{l}\text { Forward- } \\
\text { Backward }\end{array}$ & \\
\hline
\end{tabular}

For the first experiment, the data are divided into a ratio of seven to three for training and test, respectively. The 17,470 sequences are used for training, and 7,380 sequences are used for test. Each sequence is distributed randomly. The results through all experiments in this work are compared with standard BLSTM. Average accuracy rate of the proposed method is $91.11 \%$ and standard BLSTM is $89.17 \%$. For the second experiment, we group the data as generation. 18,490 sequences are used for training and approximately 2,100 sequences are used for testing each generation. Fig 2 shows that the proposed method outperforms standard BLSTM for all generations. 


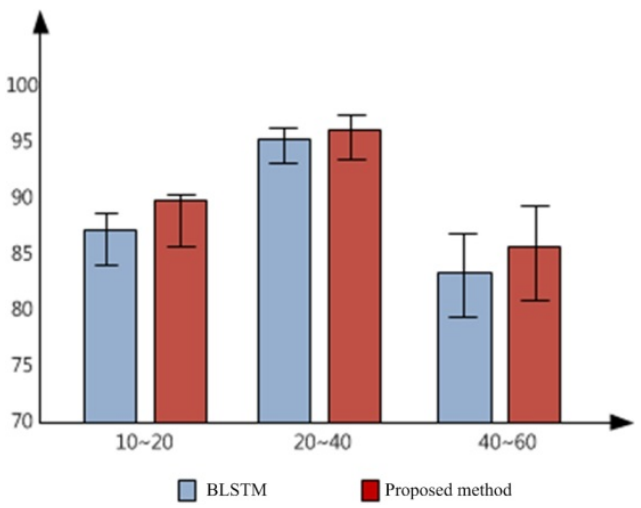

Fig. 2. The generational experiment

\section{Concluding Remarks}

In this paper, we have collected the accelerometer data from a smartphone and classified the data by using a recurrent neural network with non-gesture rejection model. Total classes of the data are twenty one including non-gestures. The performance of the standard BLSTM compared with the proposed method and our approach outperforms the standard BLSTM. However, it is not satisfactory to apply in the real world. It might be possible to obtain higher accuracy if the data are grouped with the similar meaning because some gestures have similar features. For a future work, we will investigate the characteristics of features for similar gestures.

\section{References}

1. Lane, N.D., Miluzzo, E., Lu, H., Peebles, D., Choudhury, T., Cambell, A.T.: A survey of mobile phone sensing. IEEE Communications Magazine 48(9), 140-150 (2010)

2. Chen, Q., Georganas, N.D., Petriu, E.M.: Hand gesture recognition using haar-like featuresand a stochastic context-free grammar. IEEE Trans. on Instrumentation and Measurement 57(8), 1562-1571 (2008)

3. Tran, C., Trivedi, M.M.: 3-D posture and gesture recognition for interactivity in smart spaces. IEEE Trans. on Industrial Informatics 8(1), 178-187 (2012)

4. Zhang, X., Chen, X., Li, Y., Lantz, V., Wang, K., Yang, J.: A framework for hand gesture recognition based on accelerometer and EMG sensors. IEEE Trans. on Systems, Man, and Cybernetics, Part A: Systems and Humans 41(6), 1064-1076 (2011)

5. Wheeler, K.R., Chang, M.H., Knuth, K.H.: Gesture-based control and EMG decomposition. IEEE Trans. on Systems, Man, and Cybernetics, Part C: Applications and Reviews. 36(4), 503-514 (2006)

6. Xu, R., Zhou, S., Li, W.J.: MEMS accelerometer based nonspecific-user hand gesture recognition. IEEE Sensors Journal 12(5), 1166-1173 (2012)

7. Liu, J., Wang, Z., Zhong, L., Wickramasuriya, J., Vasudevan, V.: uWave: accelerometerbased personalized gesture recognition and its applications. IEEE Int. Conf. on Pervasive Computing and Communications, 1-9 (2009) 
8. Niezen, G., Hancke, G.P.: Evaluating and optimising accelerometer-based gesture recognition techniques for mobile devices. AFRICON, 1-6 (2009)

9. Min, J.-K., Choe, B.-W., Cho, S.-B.: A selective template matching algorithm for short and intuitive gesture UI of accelerometer-builtin mobile phones. In: Cong. on Nature and Biologically Inspired Computing, pp. 660-665 (2010)

10. Marasovic, T., Papic, V.: Accelerometer-Based Gesture Classification Using Principal Component Analysis. In: Int. Conf. on Software, Telecommunications and Computer Networks, pp. 1-5 (2011)

11. Akl, A., Feng, C., Valaee, S.: A novel accelerometer-based gesture recognition system. IEEE Trans. on Signal Processing 59(12), 6197-6205 (2011)

12. Hochreiter, S., Schmidhuer, J.: Long short-term memory. Neural Computation 9(8), 1735-1780 (1997) 